\title{
Flagellar motility is required for the viability of the bloodstream trypanosome
}

\author{
Richard Broadhead ${ }^{\star \star}$, Helen R. Dawe ${ }^{2 \star}$, Helen Farr $^{2 \star}$, Samantha Griffiths ${ }^{2 \star}$, Sarah R. Hart ${ }^{3 \star}$, Neil Portman ${ }^{2 \star}$, \\ Michael K. Shaw ${ }^{2}$, Michael L. Ginger ${ }^{2}$, Simon J. Gaskell ${ }^{3}$, Paul G. McKean ${ }^{1} \&$ Keith Gull ${ }^{2}$
}

The 9+2 microtubule axoneme of flagella and cilia represents one of the most iconic structures built by eukaryotic cells and organisms. Both unity and diversity are present among cilia and flagella on the evolutionary as well as the developmental scale. Some cilia are motile, whereas others function as sensory organelles and can variously possess $9+2$ and $9+0$ axonemes and other associated structures ${ }^{1}$. How such unity and diversity are reflected in molecular repertoires is unclear. The flagellated protozoan parasite Trypanosoma brucei is endemic in sub-Saharan Africa, causing devastating disease in humans and other animals ${ }^{2}$. There is little hope of a vaccine for African sleeping sickness and a desperate need for modern drug therapies ${ }^{3}$. Here we present a detailed proteomic analysis of the trypanosome flagellum. RNA interference (RNAi)-based interrogation of this proteome provides functional insights into human ciliary diseases and establishes that flagellar function is essential to the bloodstream-form trypanosome. We show that RNAi-mediated ablation of various proteins identified in the trypanosome flagellar proteome leads to a rapid and marked failure of cytokinesis in bloodstream-form (but not procyclic insect-form) trypanosomes, suggesting that impairment of flagellar function may provide a method of disease control. A postgenomic meta-analysis, comparing the evolutionarily ancient trypanosome with other eukaryotes including humans, identifies numerous trypanosome-specific flagellar proteins, suggesting new avenues for selective intervention.

Flagellum-mediated migration between the gut and salivary glands of its tsetse fly vector is essential for progression of the trypanosome life cycle. However, the necessity for motility in an extracellular bloodstream-form trypanosome is unclear. In both forms, a single attached flagellum emerges from a posterior flagellar pocket (Fig. 1a) and comprises a membrane-bound axoneme and associated paraflagellar rod (PFR; Fig 1b). We isolated the structural axoneme and associated PFR and basal body from procyclic trypanosomes by a well-characterized procedure of detergent and high-salt treatment ${ }^{4}$. Bands and spots were cut from one- (Fig. 1c) and twodimensional gels and digested with trypsin, and the resulting peptides analysed by reverse-phase high-performance liquid chromatography coupled with tandem mass spectrometry. Bands and spots excised from ten representative gels were analysed, resulting in the identification of 522 nonredundant proteins.

Further manual mass spectrometric validation confirmed 380 proteins (see Supplementary Methods and Supplementary Fig. 1 for peptide numbers and coverage). Highly basic proteins are known to contaminate microtubule preparations owing to charge interactions ${ }^{4}$, and we noted some highly basic ribosomal proteins as recognizable contaminants. To limit contamination, we filtered the data by using an isolectric point (pI) value of 10.2 as a cut-off (the pI of the most acidic ribosomal protein), and placed 49 proteins (30 of which were ribosomal proteins) in a separate pool undoubtedly containing some genuine flagella components (for example, TbDIP13). The remaining 331 proteins constitute a T. brucei flagellum proteome ( $\mathrm{TbFP}$ ) characterized by both the inclusion of many known flagellar proteins and a lack of proteins from other subcellular compartments.

We subjected the TbFP to an in silico screen, testing which of these 331 proteins (and the pI-filtered set) were encoded in genomes of flagellated and non-flagellated eukaryotes (Supplementary Fig. 1). The TbFP is characterized by the absence of homologues in the genomes of non-flagellated eukaryotes (land plants, fungi and red algae; Fig. 1d). Shared components, such as $\alpha$ - or $\beta$-tubulin, are expected. We found that many TbFP proteins have homologues in the related trypanosomatid parasites Trypanosoma cruzi (312) and Leishmania major (286). Conservation of homologues between trypanosomatids and other flagellates reflects known structural differences among flagella of evolutionarily divergent organisms, being highest in Tetrahymena, human and Chlamydomonas (Fig. 1d). We found a much reduced proportion of homologues shared with Plasmodium, Caenorhabditis and Drosophila, which build motile or sensory cilia that are divergent in aspects of axoneme or basal body structure or formation ${ }^{5}$. We found that $208 \mathrm{TbFP}$ proteins (Fig. 1d) are trypanosomatid-specific and probably represent organism-specific flagellar structures and functions. Although these most obviously include the PFR, some are likely to be axonemal.

Several studies have sought to identify components of the eukaryotic flagellum by proteomic ${ }^{6-9}$ or bioinformatic ${ }^{10,11}$ strategies. Although highly informative, bioinformatic and comparative genomic approaches exclude genuine conserved flagella components that have homologues and orthologues in non-flagellates. Similarly, occurrence of a protein in proteomes reflects the biochemical nature of the purified material. The TbFP, the Tetrahymena thermophila ciliome $^{9}$ and the axonemal fraction of the Chlamydomonas flagellar proteome $^{8}$ are data sets derived from comparable preparations of insoluble flagellar architectures, with shared and organism-specific structures. To carry out a meta-analysis of these three data sets (Fig. 1e), we devised a stringent reciprocal BLASTP protocol that necessitated reanalysis of each data set to acquire the most current gene models available. After unification by applying the pI filter to all data sets, we undertook three reciprocal pairwise comparisons. We found that the TbFP contains the largest number of proteins in any of the three data sets. The presence of the PFR, an extra-axonemal, trypanosome-specific structure might explain the large number of proteins not encoded in the genomes of the other two organisms (indeed, the TbFP contains all of the known PFR proteins). However,

${ }^{1}$ Department of Biological Sciences, Lancaster University, Lancaster LA1 4YQ, UK. ${ }^{2}$ Sir William Dunn School of Pathology, University of Oxford, South Parks Road, Oxford OX1 3RE, UK. ${ }^{3}$ Michael Barber Centre for Mass Spectrometry, School of Chemistry, University of Manchester, Sackville Street, Manchester M60 1QD, UK.

*These authors contributed equally to this work. 
the Chlamydomonas and Tetrahymena pairwise axoneme-only comparisons revealed correspondingly large cohorts not represented in the genome of the opposing organism. Thus, the structural homogeneity of the $9+2$ axoneme seems to be dependent on a relatively few evolutionarily conserved proteins and masks considerable organism-specific elaborations that go beyond the presence of obvious extra-axonemal structures.

Present in the conserved subset are proteins with implications for human inherited diseases. Ciliary diseases are characterized by clinical presentations such as retinal degeneration, primary ciliary dyskinesia, hydrocephalus, polycystic kidney disease and polydactyly ${ }^{1}$. In addition to all three axonemal dyneins known to cause primary ciliary dyskinesia ${ }^{12}$, interrogation of the TbFP identified homologues of a further seven proteins directly implicated in diseases of human, mouse or zebrafish (Supplementary Fig. 2a). This list includes Hydin ${ }^{13}$, PACRG ${ }^{14}$ and Scorpion ${ }^{15}$; two of these, TbHydin and TbPACRG, are validated functionally here for the first time to our knowledge. We determined the human chromosomal locus for every homologue represented in the TbFP, which identified 34 genes mapping to 25 loci where diseases with a clinical spectrum suggestive of ciliary dysfunction have been mapped genetically, but where the causal gene has not been identified. We suggest that these 34 genes (Supplementary Fig. 2b) represent candidates underlying syndromes including primary ciliary dyskinesia, polycystic kidney
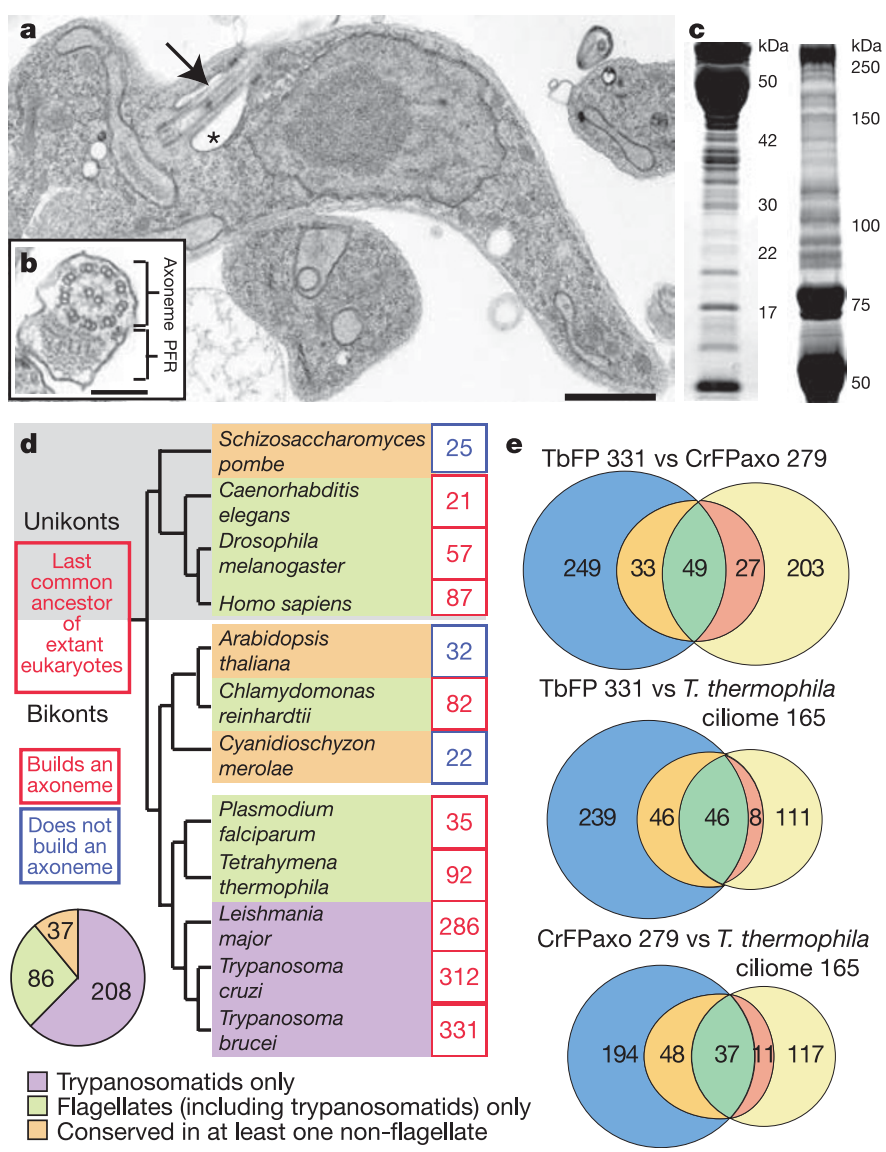

Figure 1 The T. brucei flagellar proteome. a, Transmission electron micrograph showing the flagellum (arrow) and flagellar pocket (asterisk). b, The 9+2 axoneme and the PFR. c, One-dimensional electrophoretic separation of isolated flagella. d, Distribution of TbFP protein homologues. e, Comparison of the T. brucei, Chlamydomonas reinhardtii (CrFPaxo) and T. thermophila flagellar proteomes. For each comparison, green indicates present in both; orange/red indicates present in opposing genome but not opposing proteome; blue/yellow indicates no homologue in opposing genome. Homology determined by alignment to each genome with reciprocal alignment by BLASTP. Scale bars, $1 \mu \mathrm{m}($ a); $200 \mathrm{~nm}$ (b). disease, macular and cone-rod dystrophies, retinitis pigmentosa, Rieger and BRESEK (see http://www.ncbi.nlm.nih.gov/entrez/ query.fcgi? $\mathrm{db}=\mathrm{OMIM}$ for syndrome details).

We subjected the orthologue of the cryptic hydrocephalusinducing protein Hydin to functional analysis by using inducible RNAi in procyclic trypanosomes. This resulted in a severe motility defect, revealing an impairment of ciliary function as a likely explanation for the aetiology of this disease model (Fig. 2). A further ten TbFP proteins were interrogated by this RNAi approach (Fig. 2a), eight of which (including the trypanosome-specific proteins PFR2 and HERTS) showed a flagellar phenotype on ablation (Fig. 2a). All mutants were viable except one in which flagellar detachment caused pleiotropic effects ${ }^{16}$. Two genes (TbPACRGA and TbPACRGB) comprise a gene family in T. brucei and gave no phenotype on individual ablation. Simultaneous knockdown, however, produced paralysed flagella, suggesting functional redundancy.

The high success rate of the RNAi interrogation provides experimental evidence for the integrity of the TbFP. These phenotypes extend the catalogue of trypanosome flagellar RNAi mutants, which have all been made in the procyclic form ${ }^{16-19}$, and show that motility can be severely compromised while proliferation remains unaffected. We extended our functional interrogation by carrying out RNAi against five proteins in our TbFP validation set in bloodstream-form trypanosomes (Supplementary Fig. 3a). Unexpectedly, we found that all five RNAi analyses resulted in bloodstream-form trypanosomes that did not complete cytokinesis, yielding monstrous cells with an inability to proliferate (Fig. 3 and Supplementary Fig. 3b-h). This striking contrast to the viable RNAi phenotypes produced in

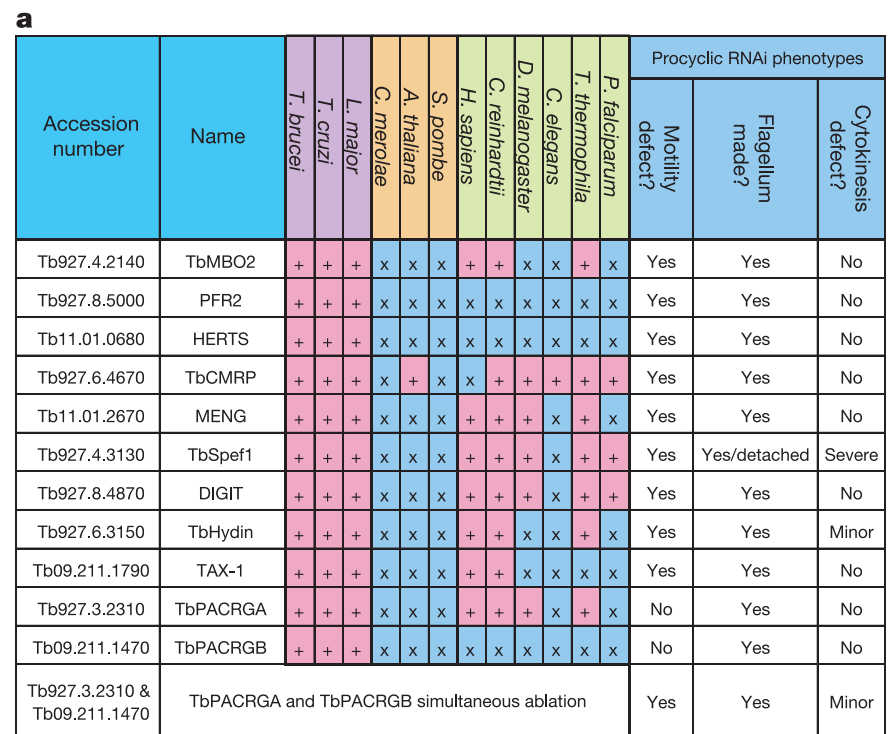

b

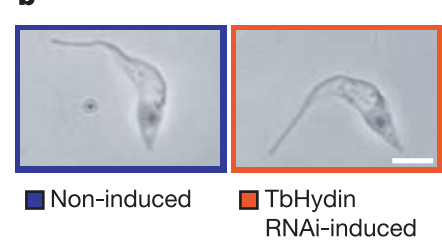

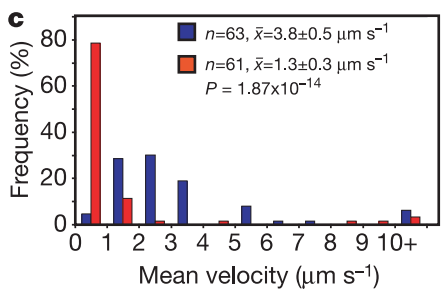

Figure 2 Biological verification of the $T$. brucei flagellar proteome. a, Biological validation of the TbFP by RNAi of procyclic trypanosomes, showing phylogenetic distribution and RNAi phenotype. ' + ' indicates presence of homologues; ' $x$ ' indicates absence of homologues. b, c, Phenotype of T. brucei Hydin (TbHydin). On RNAi induction the flagellum is built (b), but motility is severely compromised in individual cells and the cell population (c). Scale bar, $5 \mu \mathrm{m}$ (b). Blue indicates noninduced control; red indicates TbHydin RNAi-induced. $P$ value determined by Mann-Whitney $U$-test. Population statistic given as the mean \pm s.e.m. 
procyclic cells is shown in Fig. 3a-c. In each case, RNAi induction led to an inhibition of cytokinesis.

Trypanosomes have one kinetoplast (mitochondrial DNA) and one nucleus $(1 \mathrm{~K} 1 \mathrm{~N})$, and replicate these once during a cell cycle ${ }^{20}$; on RNAi induction, however, bloodstream cells ceased dividing but continued to progress through the cell cycle. Cells initiated new rounds of S phase and mitosis, leading to large contorted cells containing multiple kinetoplasts and nuclei (Fig. 3d, i, j). At $24 \mathrm{~h}$ after induction, the RNAi-induced population of 'monsters' contained large numbers of $4 \mathrm{~K} 4 \mathrm{~N}$ and $8 \mathrm{~K} 8 \mathrm{~N}$ cells (Fig. $3 \mathrm{~d}$ ).

Included in the bloodstream RNAi set were newly identified flagellar proteins such as TAX-1, TbPACRGA and DIGIT in addition to an orthologue (TbMBO2) of a Chlamydomonas protein known to regulate motility ${ }^{21}$. Given the implications of these observations, we tested whether the well-characterized procyclic snl mutant phenotype $^{17,18}$ (paralysed but viable) of the T. brucei specific PFR2 protein was different in the bloodstream form. We found that this mutant also produced a rapid, lethal and unusual phenotype with production of monstrous cells. Notably, the same construct expressed in procyclic cells reproduced the published paralysed, but viable, phenotype (Fig. 2a).

A very particular phenotype led to the death of bloodstream cells. Uninduced cells had a normal morphology (Fig. 3e), producing a new flagellum during the cell cycle before cytokinesis, with the flagellum emerging from the flagellar pocket and extending along the trypanosome. On RNAi induction, cells produced new flagella but did not complete division and lost all normal morphogenetic axes, becoming monstrously contorted and multiflagellated (Fig. $3 \mathrm{f}-\mathrm{h}$ ). Thin-section electron microscopy showed that the cell periphery was highly convoluted; multiple nuclei were present and some flagella were attached to the outside of the cell, while others were located in a highly enlarged flagellar pocket (Fig. 3i, $j$ and Supplementary Fig. 3b, c, f, g). There were also many flagella with two axonemes, which could be either parallel or antiparallel, indicative of a total loss of morphogenetic patterning (Supplementary Fig. 3d, e). These characteristics are also found in the TbMBO2 and DIGIT phenotypes (Supplementary Fig. 3f, g). Examination of the flagellar pockets indicated that although large they still showed clathrin-coated pits, suggesting that endocytotic processes were operating to some extent (for example, in the TAX-1 phenotype; Supplementary Fig. $3 \mathrm{~h}$ ).

The explanation for the lethal phenotype in bloodstream cells is that cytokinesis fails as a primary event in the absence of correct flagellar motility. Subsequent rounds of the cell cycle compound these events as new flagella and pockets are formed at inappropriate locations. The lack of precise morphogenetic axes in the resulting 'monstrous cells' leads to pleiotropic effects compromising membrane-cytoskeletal balance during morphogenesis of the pocket structure. Examination of endocytosis shows that the multiple pockets are active and can facilitate entry of antibodies and lectins. However, there is much variation in their individual capacity for vectorial internalization to nearby endomembrane compartments (Supplementary Fig. 4). An imbalance in the known high flux of endocytosis in the bloodstream form ${ }^{22}$, coupled with a focus of the secretory pathway on particular pockets in a multiflagellated cell, will lead to the large flagellar pocket phenotype as a secondary event.

In summary, we have shown that flagellum function cannot be compromised and is essential in the bloodstream trypanosome. The severity of the phenotype, its rapid onset, its lethality and its occurrence after the ablation of proteins of diverse function and location in either the axoneme or the trypanosome-specific PFR is highly significant. Coupled with our identification of a set of trypanosome-specific proteins in the TbFP, this suggests that impair-
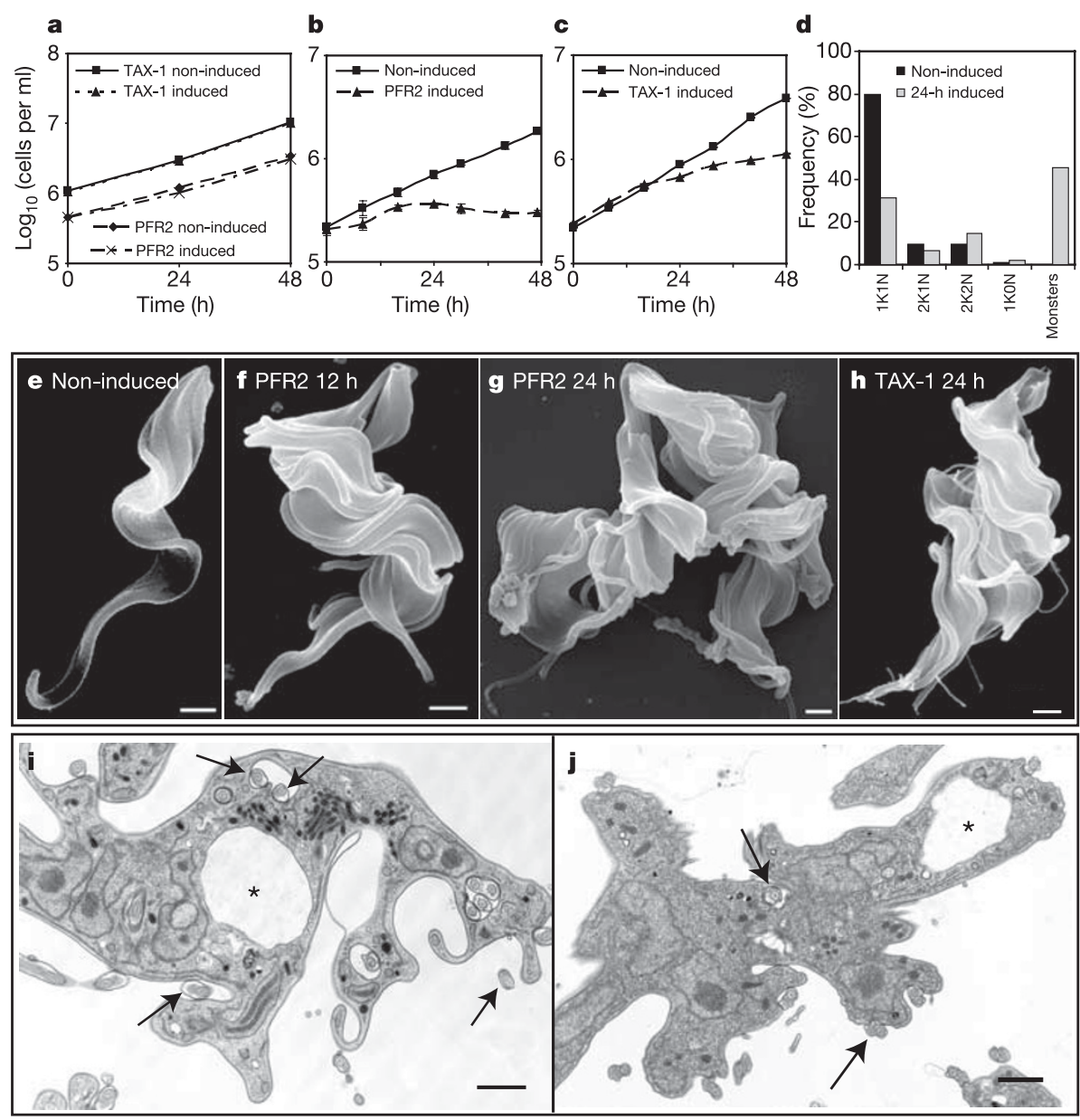

Figure 3 | Flagellar motility is essential for bloodstream trypanosomes. a-c, Ablation of PFR2 and TAX-1 affects growth in bloodstream (b, c), but not procyclic (a), forms. d, Numbers of cells with multiple nuclei and kinetoplasts increase after ablation of TAX-1 in bloodstream forms. e-h, Scanning electron micrograph showing bloodstream-form morphogenesis before (e) and after ( $\mathbf{f}-\mathbf{h})$ ablation of PFR2 and TAX-1 flagellar proteins. $\mathbf{i}, \mathbf{j}$, Transmission electron microscopy analysis of PFR2 (i) and TAX-1 (j) ablation in bloodstream forms. Arrows denote axonemes; asterisks denote flagellar pockets $(\mathbf{i}, \mathbf{j})$. Scale bar, $1 \mu \mathrm{m}$. 
ment of flagellar function may provide an avenue for disease control in African sleeping sickness.

\section{METHODS}

Preparation of flagella proteins for mass spectrometry. Procyclic T. brucei cells were collected by centrifugation $(800 \mathrm{~g}, 10 \mathrm{~min})$, washed once in PBS containing $5 \mu \mathrm{M}$ E-64d, and resuspended in PEME (100 mM PIPES, $2 \mathrm{mM}$ EGTA, $0.1 \mathrm{mM}$ EDTA and $1 \mathrm{mM} \mathrm{MgSO}_{4}$; $\mathrm{pH} 6.9$ ) containing $1 \%$ Nonidet $\mathrm{P} 40,1 \times$ Focus proteasearrest mix (Calbiochem), 7.5 $\mu \mathrm{M}$ Pepstatin A and $5 \mu \mathrm{M}$ E-64d. Cytoskeletons were collected by centrifugation and subjected to two rounds of salt extraction with PEME containing $1 \mathrm{M} \mathrm{NaCl}, 200 \mu \mathrm{g} \mathrm{ml}^{-1}$ of DNaseI, $50 \mu \mathrm{g} \mathrm{ml}^{-1}$ RNaseA, $1 \times$ Focus protease-arrest mix, $7.5 \mu \mathrm{M}$ Pepstatin A and $5 \mu \mathrm{M} \mathrm{E}-64 \mathrm{~d}$ at $4^{\circ} \mathrm{C}$ for $10 \mathrm{~min}$. Flagella pellets were collected by centrifugation $\left(16,000 \mathrm{~g}, 15 \mathrm{~min}, 4^{\circ} \mathrm{C}\right)$, washed twice in PEME and resuspended in Laemmli buffer ${ }^{23}$. Proteins from $6 \times 10^{8}$ cell equivalents were resolved by one-dimensional SDS-PAGE (using standard protocols) and two-dimensional SDS-PAGE (see Supplementary Methods) and visualized by staining with Coomassie blue. Regions, bands and spots were excised, digested with trypsin and analysed by mass spectrometry. Verification of the goodness of fit of putative matches to predicted protein sequences was done manually (see Supplementary Methods). All data that yielded information used to construct the TbFP will be uploaded to the EBI Proteomics Identification database (http://www.ebi.ac.uk/pride/), a public data repository. This resource is freely available for use by the proteomics community.

Analysis of the TbFP. A detailed description of the reciprocal BLASTP ${ }^{24}$ protocol used in comparative analysis is given in Supplementary Methods. Genetic loci containing human homologues of TbFP proteins were identified and compared to the following: first, loci implicated in known cilia-related diseases and diseases of unknown cause where the clinical presentation suggests a defect in cilia or flagella function (derived from OMIM: http://www.ncbi.nlm.nih.gov/entrez/ query.fcgi? $\mathrm{db}=\mathrm{OMIM}$ \&itool=toolbar); second, the RetNet database of retinal disorders (http://www.sph.uth.tmc.edu/Retnet/); third, human orthologues of mouse infertility model $\mathrm{s}^{25}$; and last, genes that cause polycystic kidney disease in zebrafish ${ }^{15}$.

Trypanosome cell culture and RNAi. Procyclic (427, 29-13 and derivatives) and bloodstream (90-13 and derivatives) cell lines were maintained and transfected by standard methods ${ }^{26}$. Cell growth was monitored by a CASY1 cell-counter and analyser system (Schärfe System GmbH). RNAi studies were carried out with 400-600 base-pair fragments of T. brucei coding sequence, which were amplified by PCR from genomic DNA and cloned into the inducible RNAi vector p2T7-177 (ref. 27). Primer sequences are given in Supplementary Methods. For counts of nuclei and kinetoplast number, cells were fixed in 3.6\% paraformaldehyde in PBS and dried onto microscope slides. 4,6-Diamidino-2phenylindole (DAPI) was used to visualize DNA. We counted 500 cells at each time point. Motility analyses were done essentially as described ${ }^{28}$ : time-lapse sequences were captured every $2 \mathrm{~s}$ for $40 \mathrm{~s}$ by using phase-contrast microscopy at $\times 10$ magnification and $22^{\circ} \mathrm{C}$. Mean velocities were determined for individual cells and for each cell line collectively. Statistical significance was tested by a twotailed Mann-Whitney $U$-test because samples varied in size and distributions were not suited to parametric testing. All analyses showed significance at $\alpha=0.01$ with the exception of TbPACRGA and TbPACRGB single knockdowns, which were not significant at this level.

Electron microscopy. For thin-section electron microscopy, cells were fixed in $2.5 \%$ glutaraldehyde, $2 \%$ paraformaldehyde and $0.1 \%$ picric acid in $100 \mathrm{mM}$ phosphate ( $\mathrm{pH} 6.5$ ) for $2 \mathrm{~h}$ at $4{ }^{\circ} \mathrm{C}$ followed by post-fixation in $1 \%$ osmium tetraoxide in $100 \mathrm{mM}$ phosphate buffer (pH 6.5) with $50 \mathrm{mM}$ sucrose for $1 \mathrm{~h}$ at $4{ }^{\circ} \mathrm{C}$. The fixed material was stained en bloc with $2 \%$ aqueous uranyl acetate for $2 \mathrm{~h}$ at $4^{\circ} \mathrm{C}$, dehydrated, and embedded in epon resin. For scanning electron microscopy, samples were prepared essentially as described ${ }^{29}$.

\section{Received 8 November; accepted 14 December 2005.}

1. Afzelius, B. A. Cilia-related diseases. J. Pathol. 204, 470-477 (2004).

2. Sternberg, J. M. Human African trypanosomiasis: clinical presentation and immune response. Parasite Immunol. 26, 469-476 (2004).

3. Steverding, D. \& Tyler, K. M. Novel antitrypanosomal agents. Expert Opin. Invest. Drugs 14, 939-955 (2005).

4. Robinson, D., Beattie, P., Sherwin, T. \& Gull, K. Microtubules, tubulin, and microtubule-associated proteins of trypanosomes. Methods Enzymol. 196, 285-299 (1991)

5. Briggs, L. J., Davidge, J. A., Wickstead, B., Ginger, M. L. \& Gull, K. More than one way to build a flagellum: comparative genomics of parasitic protozoa. Curr. Biol. 14, R611-R612 (2004).

6. Ostrowski, L. E. et al. A proteomic analysis of human cilia: identification of novel components. Mol. Cell. Proteomics 1, 451-465 (2002).
7. Padma, P. et al. Identification of a novel leucine-rich repeat protein as a component of flagellar radial spoke in the Ascidian Ciona intestinalis. Mol. Biol. Cell 14, 774-785 (2003).

8. Pazour, G. J., Agrin, N., Leszyk, J. \& Witman, G. B. Proteomic analysis of a eukaryotic cilium. J. Cell Biol. 170, 103-113 (2005).

9. Smith, J. C., Northey, J. G., Garg, J., Pearlman, R. E. \& Siu, K. W. Robust method for proteome analysis by MS/MS using an entire translated genome: demonstration on the ciliome of Tetrahymena thermophila. J. Proteome Res. 4, 909-919 (2005).

10. Avidor-Reiss, T. et al. Decoding cilia function: defining specialized genes required for compartmentalized cilia biogenesis. Cell 117, 527-539 (2004).

11. Li, J. B. et al. Comparative genomics identifies a flagellar and basal body proteome that includes the BBS5 human disease gene. Cell 117, 541-552 (2004).

12. Chodhari, R., Mitchison, H. M. \& Meeks, M. Cilia, primary ciliary dyskinesia and molecular genetics. Paediatr. Respir. Rev. 5, 69-76 (2004).

13. Davy, B. E. \& Robinson, M. L. Congenital hydrocephalus in hy3 mice is caused by a frameshift mutation in Hydin, a large novel gene. Hum. Mol. Genet. 12 1163-1170 (2003).

14. Lorenzetti, D., Bishop, C. E. \& Justice, M. J. Deletion of the Parkin coregulated gene causes male sterility in the quaking(viable) mouse mutant. Proc. Natl Acad. Sci. USA 101, 8402-8407 (2004).

15. Sun, Z. et al. A genetic screen in zebrafish identifies cilia genes as a principal cause of cystic kidney. Development 131, 4085-4093 (2004).

16. Moreira-Leite, F. F., Sherwin, T., Kohl, L. \& Gull, K. A trypanosome structure involved in transmitting cytoplasmic information during cell division. Science 294, 610-612 (2001)

17. Bastin, P., Ellis, K., Kohl, L. \& Gull, K. Flagellum ontogeny in trypanosomes studied via an inherited and regulated RNA interference system. J. Cell Sci. 113, 3321-3328 (2000)

18. Bastin, P., Sherwin, T. \& Gull, K. Paraflagellar rod is vital for trypanosome motility. Nature 391, 548 (1998).

19. Hill, K. L. Biology and mechanism of trypanosome cell motility. Eukaryot. Cell 2, 200-208 (2003).

20. Woodward, R. \& Gull, K. Timing of nuclear and kinetoplast DNA replication and early morphological events in the cell cycle of Trypanosoma brucei. J. Cell Sci. 95, 49-57 (1990).

21. Tam, L. W. \& Lefebvre, P. A. The Chlamydomonas MBO2 locus encodes a conserved coiled-coil protein important for flagellar waveform conversion. Cell Motil. Cytoskeleton 51, 197-212 (2002).

22. Field, M. C. \& Carrington, M. Intracellular membrane transport systems in Trypanosoma brucei. Traffic 5, 905-913 (2004).

23. Laemmli, U. K. Cleavage of structural proteins during the assembly of the head of bacteriophage T4. Nature 227, 680-685 (1970).

24. Altschul, S. F. et al. Gapped BLAST and PSI-BLAST: a new generation of protein database search programs. Nucleic Acids Res. 25, 3389-3402 (1997).

25. Liska, F. Selected genetic aspects of male infertility - what animal models tell us. Folia Biol. (Praha) 49, 129-141 (2003).

26. Wirtz, E. \& Clayton, C. Inducible gene expression in trypanosomes mediated by a prokaryotic repressor. Science 268, 1179-1183 (1995).

27. Wickstead, B., Ersfeld, K. \& Gull, K. Targeting of a tetracycline-inducible expression system to the transcriptionally silent minichromosomes of Trypanosoma brucei. Mol. Biochem. Parasitol. 125, 211-216 (2002)

28. Gadelha, C., Wickstead, B., de Souza, W., Gull, K. \& Cunha-e-Silva, N. Cryptic paraflagellar rod in endosymbiont-containing kinetoplastid protozoa. Eukaryot. Cell 4, 516-525 (2005).

29. Sherwin, T. \& Gull, K. The cell division cycle of Trypanosoma brucei brucei: timing of event markers and cytoskeletal modulations. Phil. Trans. R. Soc. Lond. B $323,573-588$ (1989).

Supplementary Information is linked to the online version of the paper at www.nature.com/nature.

Acknowledgements We thank the genome centres, particularly GeneDB at the Sanger Institute, and our laboratory colleagues, especially S. Gordon and P. Taylor, for access to unpublished data and constructive discussions. This work was funded by the Biotechnology and Biological Sciences Research Council, the Wellcome Trust, the Royal Society and the Edward P. Abraham Trust. M.L.G. is a Royal Society University Research Fellow; K.G. is a Wellcome Trust Principal Research Fellow.

Author Contributions The first six authors contributed equally to this work and are listed alphabetically. Individual contributions were as follows: R.B. proteomics and bioinformatic analysis; H.R.D. proteomics, bioinformatic and functional analyses; H.F. and S.G. functional analysis; S.R.H. proteomics; N.P. proteomics, bioinformatic and functional analyses.

Author Information Reprints and permissions information is available at npg.nature.com/reprintsandpermissions. The authors declare no competing financial interests. Correspondence and requests for materials should be addressed to K.G. (keith.gull@path.ox.ac.uk). 